\title{
Prevalencia de hallazgos compatibles con material de osteosíntesis en radiografías panorámicas
}

\author{
Dayana Herrera A', Verónica Verdugo $T^{2}$.
}

1. Odontóloga del MSP de Ecuador, Ecuador.

2. Docente de la Universidad Católica de Cuenca (UCACUE), Ecuador.

Prevalence of findings compatible with osteosynthesis material in panoramic radiographs

Objective: To determine the prevalence of findings compatible with osteosynthesis material in panoramic radiographs, at the radiological center of the Catholic University of Cuenca, in the period 2015-2016. Materials and methods: the methodology used was with a quantitative, descriptive, observational and retrospective approach. The population for this study consisted of the whole collection of panoramic radiographs deposited in the database of the Catholic University of Cuenca, Faculty of Dentistry, radiological center in the period 2015 - 2016, totaling 3243 radiographs, from which were excluded digital panoramic radiographs that were taken outside the focal section, radiographs taken with an inadequate technique, cephalic radiographs, occlusal radiographs and digital radiographs that were found repeated in the database or with incomplete patient data that could not define the age or sex to which they corresponded, giving a total of 117 excluded digital radiographs. Results: it was shown that there is a minimal prevalence of radiographic findings compatible with osteosynthesis materials in the radiological center of the Catholic University of Cuenca, represented by $0.2 \%$ corresponding to 7 patients, being more prevalent in the mandible and upper maxilla in equal proportion and being less frequent in the mandibular condyle, represented by $0.03 \%$. All these studies showed that it presented in patients of average age 34 years, with a standard deviation of 15 years; however, no significant difference was found between males and females.

Keywords: Findings, Osteosynthesis, Materials, Radiographics.

Resumen. Objetivo: Determinar la prevalencia de hallazgos compatibles con material de osteosíntesis en radiografías panorámicas, en el centro radiológico de la Universidad Católica de Cuenca en el periodo 2015 - 2016. Materiales y métodos: la metodología utilizada fue con un enfoque cuantitativo, descriptivo, observacional y retrospectivo. La población de este estudio se conformó por todo el universo de las radiografías panorámicas depositadas en la base de datos del centro radiológico de la Universidad Católica de Cuenca, Facultad de Odontología en el periodo 2015 - 2016 que suman un total de 3.243 radiografías, de las cuales fueron excluidas las radiografías panorámicas digitales que se han tomadas fuera del corte focal, radiografías tomadas con una técnica inadecuada, radiografías cefálicas, radiografías oclusales y radiografías digitales que se encontraron repetidas en la base de datos o con datos incompletos del paciente que no se pueda definir la edad o el sexo al que corresponden, siendo un total de 117 radiografías digitales excluídas. Resultados: se evidencio que existe una mínima prevalencia de hallazgos radiográficos compatibles con materiales de osteosíntesis en el centro radiológico de la Universidad Católica de Cuenca, representado con un $0,2 \%$ correspondiente a 7 pacientes, siendo más prevalente en la mandíbula y maxilar superior en igual proporción y siendo menos frecuente en el cóndilo mandibular representado por un $0,03 \%$. Todos estos estudios arrojaron que se presentaba en paciente de 34 años de edad promedio, con una desviación estándar de 15 años; sin embargo, no se encontró diferencia significativa entre los dos sexos antes mencionados.

Palabras clave: Hallazgos, Osteosíntesis, Materiales, Radiográficos.

Herrera $D$, et al. Prevalencia de hallazgos compatibles con material de osteosíntesis en radiografías panorámicas. Rev Chil Radiol 2017; 23(4): 156-163.

Correspondencia: Dayana Herrera / dayaceherrera@hotmail.com.

Trabajo enviado el 21 de noviembre de 2017. Aceptado para publicar el 09 de diciembre de 2017. 


\section{Introducción}

La osteosíntesis es un procedimiento quirúrgico que nos permite estabilizar y fijar fragmentos óseos mediante implantes metálicos que entran en contacto directo con el hueso (fijación interna) respetando las reglas biomecánicas y biológicas, devolviendo la funcionalidad lo más rápido posible, para lograr su consolidación con una recuperación del paciente a su vida habitual ${ }^{1-3}$.

Como menciona Mauricio Muller que en la década de 1960, establecieron reglas básicas de la osteosíntesis con placa y definieron las modalidades quirúrgicas, pero con la aparición de los nuevos implantes se ha modificado totalmente dicha filosofía y su aplicación práctica ${ }^{4}$.

Dentro del área de radiología los hallazgos radiológicos relacionados con trauma, cirugía ortognática o reconstructiva pueden ser determinados por la presencia de placas de osteosíntesis que permiten una fijación segura y la cicatrización de los fragmentos ${ }^{5}$.

La radiografía panorámica es uno de los exámenes más utilizados para realizar diagnósticos en dientes y maxilares (Dryland \& Ellis, 1990) y es de amplio uso en el campo odontológico tanto por su costo, sensibilidad y especificidad, siendo necesario establecer parámetros para una correcta interpretación y posterior diagnóstico presuntivo o definitivo y además como un respaldo frente a todas las implicancias médico-legales ${ }^{5-7}$.

Sin embargo, en un estudio realizado en el 2013 por Alqerban y col, los observadores llegaron a un mismo acuerdo el cual mencionaron que tenían un nivel significativamente más alto de confianza en sus planes de tratamiento quirúrgicos basados en imágenes 3D que en sus planes basados en imágenes 2D, pero sin embargo, este tipo de estudio aún se encuentra muy limitado debido a su gran costo por lo que los profesionales deciden hacer una radiografía panorámica por la comodidad del paciente ${ }^{8,9}$.

En este estudio se utilizó radiografías panorámicas digitales con adecuada densidad y contraste, seleccionando imágenes en las que se observen la presencia de placas, mallas de reconstrucción, torniIlos, alambres y de esta manera poder determinar la prevalencia de los hallazgos radiográficos compatibles con materiales de osteosíntesis.

La incidencia y etiología de las fracturas maxilofaciales por las que es necesaria la colocación de materiales de osteosíntesis varía ampliamente entre los diferentes países de acuerdo a sus factores, incluyendo los ambientales, factores culturales y socioeconómicos. Las causas principales de todo el mundo son los accidentes de tráfico, agresiones, caídas y lesiones deportivas. Estudios realizados en países como Singapur, Nueva Zelandia, Dinamarca, Japón, la región del Oriente Medio, han demostrado que los accidentes en automóvil son la causa más común de fracturas maxilofaciales, seguido de traumatismos por accidentes laborales, accidentes deportivos y violencia interpersonal que por lo general se da por el abuso del alcohol y la creciente agresión en la sociedad en la forma de peleas, asaltos y disparos ${ }^{10,11}$. En cambio en Ecuador se determinó que las causas más comunes para las fracturas maxilofaciales varía dependiendo de la ciudad en la que se encuentren como es el ejemplo del Hospital Enrique Garcés de Quito que la principal causa son las agresiones físicas con un $41 \%^{12}$, Hospital Luis Vernaza de Guayaquil son por accidentes de tránsito por motocicleta $(61.8 \%)^{13} \mathrm{y}$ de automóvil con un (16.9\%), en Hospital Isidro Ayora de Loja y el Hospital Vicente Corral Moscoso de Cuenca es por accidentes automovilísticos con un $34.78 \%$ y un $46,8 \%$ respectivamente ${ }^{14,15}$.

El presente estudio tiene por objetivo determinar la prevalencia e identificación correcta de MO (Materiales de Osteosíntesis), analizando toda la base de datos de radiografías panorámicas digitales del centro radiológico de la Universidad Católica de Cuenca en los años 2015 - 2016, de la misma manera poder determinar la zona anatómica facial que presenta $\mathrm{MO}$ y el sexo y la edad de mayor prevalencia. Esto con el fin de reportar y brindar una fuente de información trascendental para orientar a los alumnos y profesionales del área de salud, principalmente en el área de odontología, acerca de cuan frecuentes son los pacientes que acuden a nuestra consulta odontológica con materiales compatibles de osteosíntesis, de la misma manera brindar a la sociedad un nuevo aporte sobre dicha prevalencia; en vista que hasta la actualidad a nivel de todo el Ecuador, no existe un estudios referentes con el presente tema establecido. Por lo que ayudaría a tener una base científica irrefutable para futuras investigaciones y de esta manera hacer una inferencia estadística ${ }^{11}$.

\section{Metodología}

El enfoque de la investigación es cuantitativo, el diseño y nivel de investigación es descriptivo, el tipo de investigación es documental, observacional y retrospectivo. La población está formada por todo el universo de las radiografías panorámicas depositadas en la base de datos del centro radiológico de la Universidad Católica de Cuenca, Facultad de Odontología en el periodo 2015 - 2016 que suman un total de 3.243 radiografías, fueron excluidas del estudio las radiografías panorámicas digitales que presenten algún tipo de error o incoherencias como radiografías tomadas fuera del corte focal, con una técnica inadecuada, radiografías cefálicas, oclusales y radiografías digitales que se encontraron repetidas en la base de datos o con datos incompletos del paciente que no se pueda definir la edad o el sexo al que corresponden, siendo un total de 117 radiografías digitales excluídas. 
Para que esta investigación se lleve a cabo se solicitó un permiso al Coordinador del Centro Radiológico de la Universidad Católica de Cuenca, posterior al permiso de acceso se procedió a realizar una calibración por parte del experto; la toma de datos se la realizo desde el programa SIDEXIS donde se fueron analizando cada una de las 3.243 radiografías panorámicas, finalmente todos los datos necesarios para el estudio se depositaron en una base de datos digital elaborada en tablas de Excel.

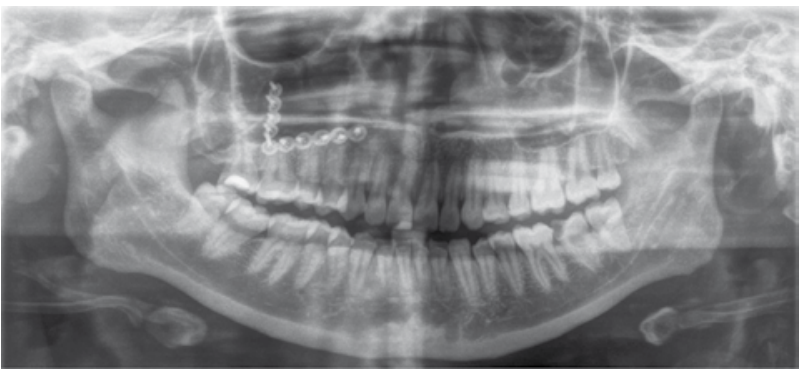

Figura 2. Posible fractura facial.

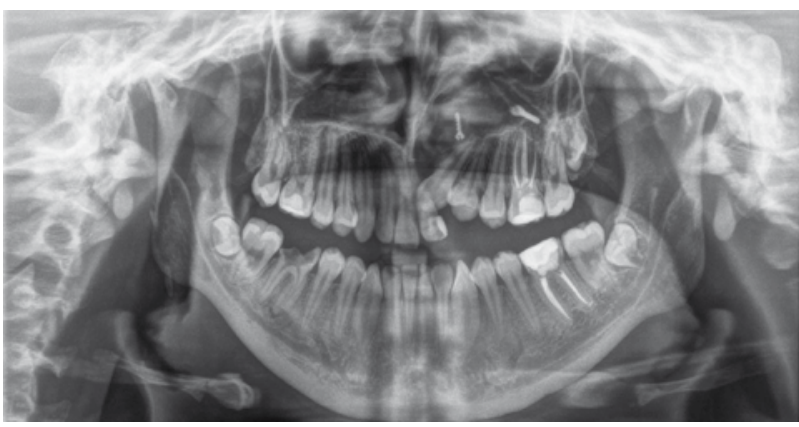

Figura 3. Reconstrucción maxilar en paciente de sexo masculino.

Tabla 1. Prevalencia total de hallazgos radiográficos compatibles con materiales de osteosíntesis.

\begin{tabular}{|lcc|}
\hline Prevalencia & $\mathbf{n}$ & $\%$ \\
\hline No tiene & 3119 & 99,8 \\
Sí tiene & 7 & 0,2 \\
Total general & 3126 & 100 \\
\hline
\end{tabular}

Tabla 2. Prevalencia de hallazgos radiográficos compatibles con materiales de osteosíntesis según el sexo.

\begin{tabular}{|lccccccc|}
\hline Materiales de osteosíntesis & \multicolumn{3}{c}{ Sexo } & \multicolumn{2}{c|}{} \\
& \multicolumn{2}{c}{ Femenino } & Masculino & \multicolumn{2}{c|}{ Total general } \\
& $\mathbf{n}$ & $\%$ & $\mathbf{n}$ & $\%$ & $\mathbf{n}$ & $\%$ \\
\hline No tiene & 1921 & 99,8 & 1198 & 99,8 & 3119 & 99,8 \\
Sí tiene & 4 & 0,2 & 3 & 0,2 & 7 & 0,2 \\
Total general & 1925 & 100 & 1201 & 100 & 3126 & 100 \\
\hline & & & & & \multicolumn{2}{c}{ Chi cuadrado $p=0.81$} \\
\hline
\end{tabular}

Tabla 3. Hallazgos radiográficos compatibles con osteosíntesis según la edad promedio y desviación estándar.

\begin{tabular}{|lcc|}
\hline $\begin{array}{l}\text { Materiales de } \\
\text { Osteosíntesis }\end{array}$ & $\begin{array}{c}\text { Promedio } \\
\text { de edad }\end{array}$ & $\begin{array}{c}\text { Desvest de } \\
\text { edad }\end{array}$ \\
\hline No tiene & 37 & 25 \\
Sí tiene & 34 & 15 \\
Total general & 35,5 & 25,5 \\
\hline
\end{tabular}


Tabla 4. Hallazgos radiográficos compatibles con materiales de osteosíntesis según la edad promedio y desviación estándar dependiendo al sexo que se presenta.

\begin{tabular}{|c|c|c|c|c|c|c|}
\hline \multicolumn{7}{|c|}{ Materiales de osteosíntesis } \\
\hline & \multicolumn{2}{|c|}{$\begin{array}{l}\text { Promedio de edad } \\
\text { Femenino } \quad \text { Masculino }\end{array}$} & \multicolumn{2}{|c|}{$\begin{array}{l}\text { Desvest de edad } \\
\text { Femenino Masculino }\end{array}$} & $\begin{array}{l}\text { Total } \\
\text { Promedio } \\
\text { de edad }\end{array}$ & $\begin{array}{l}\text { Total } \\
\text { Desvest } \\
\text { de edad }\end{array}$ \\
\hline No tiene & 38 & 35 & 28 & 20 & 37 & 25 \\
\hline Sí tiene & 40 & 27 & 15 & 13 & 34 & 15 \\
\hline Total general & 39 & 31 & 28,2 & 34,7 & 35 & 25,5 \\
\hline
\end{tabular}

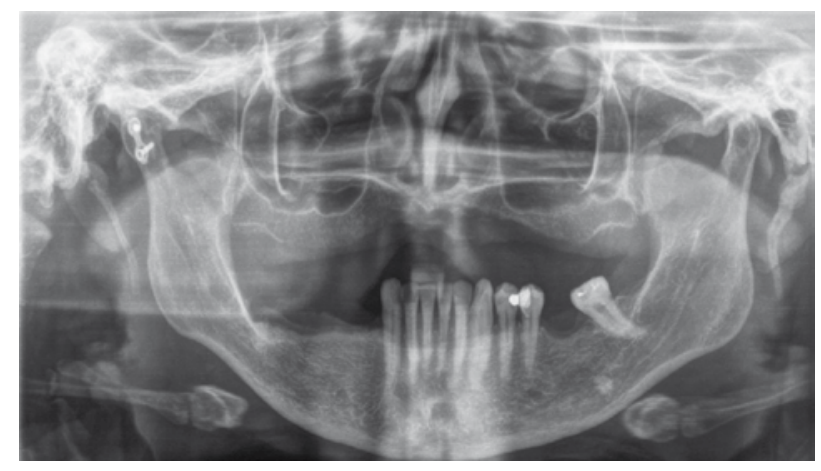

Figura 4. Fractura condilar derecha.

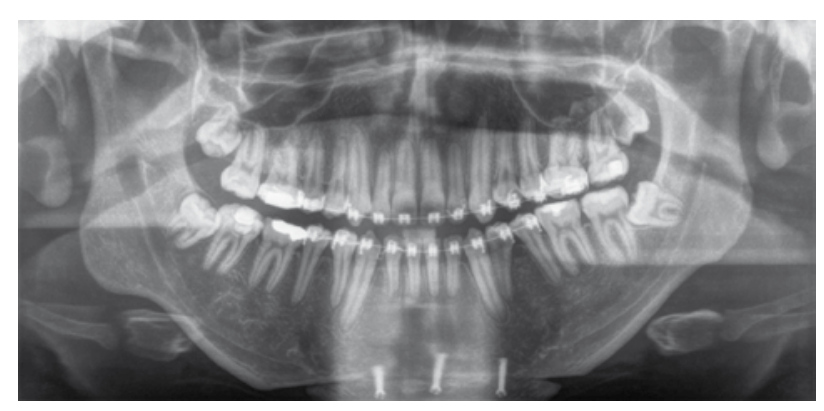

Figura 5. Tratamiento de cirugía de mentón.

Tabla 5. Hallazgos radiográficos compatibles con materiales de osteosíntesis de acuerdo a la localización anatómica.

\begin{tabular}{|c|c|c|c|c|c|c|c|c|}
\hline \multicolumn{9}{|c|}{ Material de osteosíntesis } \\
\hline \multicolumn{3}{|c|}{$\begin{array}{l}\text { Material de osteosíntesis } \\
\qquad \begin{array}{c}\text { Maxilar } \\
\text { Superior }\end{array}\end{array}$} & \multicolumn{2}{|c|}{$\begin{array}{c}\text { Cuerpo } \\
\text { Mandibular }\end{array}$} & \multicolumn{2}{|c|}{$\begin{array}{l}\text { Sínfisis } \\
\text { Mandibular }\end{array}$} & \multicolumn{2}{|c|}{ Cóndilo } \\
\hline & $\mathbf{n}$ & $\%$ & $\mathbf{n}$ & $\%$ & $\mathbf{n}$ & $\%$ & & $\%$ \\
\hline Presenta & 3 & 0,1 & 3 & 0,1 & 2 & 0,06 & 1 & 0,03 \\
\hline No presenta & 3123 & 99,9 & 3123 & 99,9 & 3124 & 99,94 & 3125 & 99,97 \\
\hline \multirow[t]{2}{*}{ Total general } & 3126 & 100 & 3126 & 100 & 3126 & 100 & 3126 & 100 \\
\hline & & & & & & \multicolumn{3}{|c|}{ Chi cuadrado $p=0.75$} \\
\hline
\end{tabular}


Tabla 6. Hallazgos radiográficos compatibles con materiales de osteosíntesis en todas las partes de la mandíbula según el sexo en el que se presenta.

\begin{tabular}{|c|c|c|c|c|c|c|}
\hline \multirow[t]{3}{*}{$\begin{array}{l}\text { Localizacion } \\
\text { toda la mand }\end{array}$} & \multicolumn{4}{|c|}{ Sexo } & \multirow{2}{*}{\multicolumn{2}{|c|}{ Total }} \\
\hline & \multicolumn{2}{|c|}{ Femenino } & \multicolumn{2}{|c|}{ Masculino } & & \\
\hline & $\mathrm{n}$ & $\%$ & & $\%$ & $\mathbf{n}$ & $\%$ \\
\hline Presenta & 3 & 0,16 & 2 & 0,17 & 5 & 0,2 \\
\hline No presenta & 1922 & 99,84 & 1199 & 99,83 & 3121 & 99,8 \\
\hline Total general & 1925 & 100 & 1201 & 100 & 3126 & 100 \\
\hline
\end{tabular}

\section{Resultados}

Se puede evidenciar que existe una mínima prevalencia de hallazgos radiográficos compatibles con materiales de osteosíntesis (Figura 1) en el Centro Radiológico de la Universidad Católica de Cuenca, representado con un $0,2 \%$ correspondiente a 7 pacientes (Tabla 1); al comparar la frecuencia estadística entre el sexo femenino (Figura 2) y masculino (Figura 3 ), no se encontró diferencia significativa (Tabla 2) y como promedio de edad en general en la que se presentan los materiales de osteosíntesis esta entre los 34 años de edad (Tabla 3), pero según el sexo de los pacientes se pudo observar que los promedios de edades están entre los 40 y 27 años respectivamente del sexo Femenino y Masculino (Tabla 4). Según la posición anatómica donde se presentan dichos materiales de osteosíntesis se observó que existe menor prevalencia en el cóndilo mandibular (Figura 4), representada con $0,03 \%$, seguida de la sínfisis mandibular (Figura 5) y en igual proporción en el maxilar superior y cuerpo mandibular (Figura 6) (Tabla 5), se analizó únicamente las partes de la mandíbula y se determinó que no existe diferencia alguna entre el sexo femenino del masculino (Tabla 6).

\section{Discusión}

Existe una mínima prevalencia de hallazgos radiográficos compatibles con materiales de osteosíntesis en el Centro Radiológico de la Universidad Católica de Cuenca, representado con un $0,2 \%$ de una muestra de 3.126 radiografías, nuestros valores son menores en comparación a un estudio realizado en Colombia 2016 por Alvarado y cols, ya que la muestra de ellos fue de 10.000 radiografías obteniendo un 1,7\% de prevalencia de estos hallazgos ${ }^{5}$.

En cuanto a la edad promedio en la que existe mayor prevalencia de estos hallazgos radiográficos o de pacientes con traumatismos maxilofaciales que han sido tratados quirúrgicamente colocando materiales de osteosíntesis tenemos que tanto en estudios realizados en una población de Colombia 2016 (33.8 años) $)^{5}$,de Ámsterdam 2012 (35,9 años) ${ }^{10}$, de Vizcaya - España 2009 (42,4 años) ${ }^{16}$, del Hospital Hernán Henríquez Aravena de Temuco de Chile 2011, Hospital Enrique Garcés de Quito y el Hospital Vicente Corral Moscoso (20 - 29 años) ${ }^{12,15,17}$ y nuestro estudio tienen una relevante coincidencia en cuanto a las edades ya que el nuestro análisis arrojo un promedio de 37 años de edad con una desviación estándar de \pm 25 años; sin embargo se encontraron estudios realizados al sur de Brasil 2016 por Quevedo ${ }^{11}$ y en Loja - Ecuador por Oñate ${ }^{14}$ que nos mencionan la prevalencia de pacientes ingresados en el hospital de emergencias por traumatismos maxilofaciales y que fueron tratados quirúrgicamente a través de materiales de osteosíntesis es mayor en niños y adolescentes que en adultos, esto está dado a que la causa principal para la fractura maxilofacial fueron accidentes como las caídas y la violencia interpersonal.

De la misma manera tenemos un estudio realizado por Garcia A en Barcelona 2011 ${ }^{18}$, de los materiales de osteosíntesis localizados únicamente a nivel mandibular, los cuales obtuvieron resultados de una población del $100 \%$ correspondientes al sexo masculino con promedio de edad de 27,03 años (rango 18 - 40) coincidiendo exactamente con nuestro estudio ya que a nivel mandibular también fue mayor en el sexo masculino y con una edad promedio de 27 años.

Al comparar la frecuencia estadística entre el sexo femenino y masculino, no se encontró diferencia significativa, coincidiendo con el estudio de Van den Bergh $B, 2012^{10}$, aunque relativamente se podría decir que existe mayor prevalencia en el sexo masculino ya que se obtuvo un mismo porcentaje que en el sexo femenino en una menor cantidad de radiografías analizadas, coincidiendo con los estudios realizados en Brasil 2016 con un $65,2 \%{ }^{11}$, Chile 2011 representado con un $91,7 \%{ }^{17}$ y Ecuador con un promedio de $83,4 \%$ a nivel de las 4 ciudades principales $^{12,13,14}$; presentándose todo lo contrario, en 
el estudio realizado en Bogotá - Colombia ${ }^{5}$ los cuales obtuvieron un $60,1 \%$ de hallazgos de materiales de osteosíntesis en el sexo femenino, esto se debe a que en todos los estudios mencionados anteriormente estaban relacionados con traumatismos siendo la causa principal para la colocación de materiales de osteosíntesis en el sexo masculino, a diferencia en el estudio de Bogotá - Colombia la mayoría de pacientes analizados eran compatibles con cirugías ortognáticas dada por anomalías dentofaciales.

La distribución anatómica del trauma donde se colocaron materiales de osteosíntesis según el estudio realizado por Alvarado y cols en el 2016, nos emite los siguientes resultados donde el lugar menos frecuente es el cóndilo combinado con el cuerpo mandibular y el más frecuente es la región nasal y del maxilar superior, coincidiendo con el estudio realizado por Quevedo 2016, Oñate en el 2012 con 35\%, Molina en el 2005 con un $13,4 \%$ quienes menciona que esto se debe a la prominente posición anatómica y la fragilidad que poseen los huesos nasales, coincidiendo elocuentemente con nuestro estudio donde obtuvimos mayor prevalencia en maxilar y cuerpo mandibular en las mismas proporciones con un $0,1 \%$ y en el cóndilo con un $0,03 \%$. De las 4 ciudades principales a nivel de Ecuador se evidencio que existe una gran disputa entre las fracturas mandibulares y fracturas órbitocigomáticas en una relación de 2:2; siendo de fracturas mandibulares en la ciudad de Guayaquil con un 30,2\% y Quito con un 32\%; fracturas órbitocigomáticas en las ciudades de Cuenca con un 34\% y Loja con un $35 \%$.

Contradiciendo en su totalidad a nuestro análisis son los estudios realizados en 579 pacientes de Ámsterdam - $2012^{10}$ donde la fractura con materiales de osteosíntesis más frecuente es la combinación de cuerpo y cóndilo mandibular representado con un $26,8 \%$ y el estudio de 53 pacientes del Hospital Universitario Sant Joan D'Alacant, Alicante, España $2012^{19}$, donde la fractura más frecuente fue tanto del cuello del cóndilo como subcondíleas representando un $55 \%$, esto se debe que los dos estudios mencionados anteriormente fueron hechos en un grupo de pacientes con fracturas maxilofaciales y fracturas del proceso condilar; razón por la cual, arroja datos elevado en la región condilar en comparación con nuestro estudio que fue realizado en una muestra tanto de pacientes sanos como de pacientes con fracturas maxilofaciales.

\section{Conclusión}

Existe una baja prevalencia de hallazgos radiográficos compatibles con osteosíntesis representado con un $0,2 \%$ a pesar que la muestra fue relativamente amplia, esto puede deberse al tipo de pacientes manejados y atendidos en la Facultad de Odontología de la Universidad Católica de Cuenca, los cuales son pacientes odontológicos de baja y moderada complejidad.

\section{Referencias}

1. urv.cat [Internet]. Terragona: URV Solidaria [citado 30 abril 2017]. Disponible en: http://www.urv.cat/ media/upload/arxius/URV_Solidaria/COT/Contenido/ Tema_2/2.5._tecnicas_de_tratamiento_de_las_fracturas.pdf.

2. Ceballos A, Balmaseda R, Rivero J, Pedroso M. Osteoporosis y osteosíntesis. Rev Cubana Ortop Traumatol [Internet] 2012 Jun [citado 2017 abril 04] ; 26(1): 98-108. Disponible en: http://scielo. sld.cu/scielo.php?script=sci_arttext\&pid $=$ S0864 215X2012000100010\&lng=es.

3. Ramos E, García F, Domínguez C, Chávez G, Meza G, Buffo I. Principios biomecánicos para la osteosíntesis, re-evolución. Medigraphic [Internet] 2016 [citado 2017 May 02]; 30(1): s1-s8. Disponible en: http://www. medigraphic.com/pdfs/ortope/or-2016/ors161a.pdf

4. Cognet J, Altman M, Simon P. Material de osteosíntesis: Tornillos y placas. elsevier [Internet]. 2008 octubre [citado 2017 mayo 24]; 1(1): 1-10. Disponible en: http://www.sciencedirect.com/science/article/pii/ S2211033X09716032

5. Alvarado E, Chanis J, Barrientos S, Rodríguez A. Hallazgos compatibles con material de osteosíntesis en 10.000 radiografías panorámicas: Un estudio descriptivo en Bogotá, Colombia. Scielo [Internet] 2016 Mayo [citado 2017 may 02]; 18(27): 34-41. Disponible en: http://www.scielo.edu.uy/pdf/ode/v18n27/ v18n27a05.pdf

6. Rodríguez B, Beltrami R, Tagliabo A, Rizzo S, Lupi S. Diferencias entre panorámica y Cone Beam-CT en la evaluación quirúrgica de terceros molares inferiores. Journal of Clinical and Experimental Dentistry [Internet] 2017 febrero [citado 2017 may 19]; 9(2): e259-e265. Disponible en: https://www.ncbi.nlm.nih.gov/pmc/ articles/PMC5303328/.

7. Zapata S, Medina H, Sarabia D, Navarro P, Olate S. Análisis Morfométrico de la Mandíbula de Pacientes con Asimetría Facial Asociada a Hiperplasia Condilar. Estudio en Radiografía Panorámica. SCIELO [Internet] 2014 marzo [citado 2017 mayo 05]; 32(1): 161-165.Disponible en: http://www.scielo.cl/scielo.php?script=sci_ arttext\&pid=S0717-95022014000100028\&Ing =es\& $n$ $\mathrm{rm}=\mathrm{iso} \& \mathrm{tlng}=\mathrm{es}$

8. Alqerban A, Hedesiu M, Baciut M, Nackaerts O, Jacobs $R$, Fieuws $S$, et al. La planificación del tratamiento Pre-quirúrgica de retenciones canino superior utilizando panorámica vs formación de imágenes CT de haz cónico. Radiología Dentomaxilofacial 2013 [citado 2017 May 19]; 42 (9): 20130157. Disponible en: https:// www.ncbi.nlm.nih.gov/pmc/articles/PMC3828021/

9. Camarena A, Gonzales E, Cruzado L, Liñan C. Métodos de diagnóstico imagenológico para optimizar el plan de tratamiento y pronóstico de caninos maxilares. Scielo [Internet] 2016 octubre [citado 2017 mayo 09]; 26 (4): 263-270. Disponile en: http://www.scielo. org.pe/scielo.php?script $=$ sci_arttext\&pid $=$ S1019$43552016000400009 \& \operatorname{lng}=$ en \& $n r m=$ iso

10. Van den Bergh B, Karagozoglu KH, Heymans MW, Forouzanfar T. Aetiology and incidence of maxillofacial 
trauma in Amsterdam: A retrospective analysis of 579 patients. Journal of Cranio-Maxillo-Facial Surgery [Internet] 2012 septiembre [citado 20172 mayo 05]; 40(6): e165-e169. Disponible en: https://www. clinicalkey.es/\#!/content/playContent/1-s2.0-S1010 518211001909 ? returnurl=http:\%2F\%2Flinkinghub. elsevier.com\%2Fretrieve\%2Fpii\%2FS10105182110 01909\%3Fshowall\%3Dtrue\& referrer=https:\%2F\%2 Fwww.ncbi.nlm.nih.gov\%2F

11. Quevedo S, Borges T, Becker L, Castilhos M, González P, Floriani P. A Retrospective Study of Oral and Maxillofacial Injuries in an emergency Hospital in Southern Brazil. Pesquisa Brasileira em Odontopediatria e Clínica Integrada [Internet] 2016 septiembre [citado 2017 mayo 25]; 16(1): 339-350. Disponible en: http://www.redalyc.org/articulo.oa?id=63749588036

12. Paredes W. "Etiología de las fracturas maxilofaciales en los pacientes atendidos en el Hospital Enrique Garcés de Quito. Período de enero a julio del 2012 [Internet]. 1ra ed. Dspace; 2012 [citado 2017 agosto 01]. Disponible en: http://www.dspace.uce.edu.ec/ bitstream/25000/485/1/T-UCE-0015-29.pdf

13. Méndez F, Encalada D, Torres M. "Prevalencia del Traumatismo Maxilofacial en el Hospital Luis Vernaza, Periodo Enero a Diciembre del 2010". Rev Med HJCA [Internet] 2012 [citado 2017agosto 01]; 4(2): 123 - 127. Disponoible en: file://C:/Users/usuario/ Downloads/230-791-1-PB.pdf.

14. Oñate C, Oñate D, Granda A, Arias C. "Análisis Epidemiológico de Trauma Maxilofacial". Rev Med de Nuestros Hospitales [Internet] 2012 [citado 2017 julio 31]; 18(1): 14-19. Disponible en: http://www. revistamedica.org.ec/docs/rmnh/2012/vol18/revistamedica_vol18_no1.pdf

15. Astudillo R, Salamea J, Crespo P, Salamea P. "Trauma, diez años de experiencia Hospital Vicente Corral Moscoso". Rev de la facultad de ciencias médicas [Internet] 2010 [citado 2017 agosto 03]: 2330. Disponible en: http://dspace.ucuenca.edu.ec/ bitstream/123456789/19963/1/Rub\%C3\%A9n\%20 Astudillo.pdf

16. Muñoz J, García J, Gabilondo F. Organización en el tratamiento del traumatismo panfacial y de las fracturas complejas del tercio medio. Cir plást iberolatinoam [Internet] 2009 Mar [citado 2017 mayo 24]; 35(1): 43-54. Disponible en: http://scielo.isciii. es/scielo.php?script $=$ sci_arttext $\&$ pid $=$ S037678922009000100010\&lng $=\bar{e}$.

17. Martínez F, Unibazo A, Almeida A, Alister J, Olate S. Estudio Descriptivo del Sistema Hospitalario en Relación a Pacientes Sometidos a Tratamiento Quirúrgico por Trauma Facial en el Hospital Hernán Henríquez Aravena de Temuco, Chile. Int J Odontostomat [Internet]. 2011 Ago [citado 2017 mayo 22]; 5(2): 141-146. Disponible en: http://www. scielo.cl/scielo.php?script=sci_arttext\&pid=S0718381X2011000200005\&lng=es. http://dx.doi.org/10.4067/ S0718-381X2011000200005.

18. García A. Comparación en el tratamiento de las fracturas de ángulo mandibular mediante osteosíntesis con una miniplaca versus dos miniplacas [Internet] 1ra ed. Barcelona: Dipòsit Digital de Documents de la UAB; 2011 [actualizado 2017 de enero 26; citado 2017 abril 21]. disponible en: http://ddd.uab.cat/pub/ trerecpro/2011/hdl_2072_171750/TR_DePabloGarciaCuenca.pdf

19. Goizueta C, Pastor D, Agüero E, Sebastián C. Osteosíntesis intraoral asistida por endoscopia en las fracturas del proceso condilar de la mandíbula: Revisión de 53 casos. Rev Esp Cirug Oral y Maxilofac [Internet] 2012 Dic [citado 15 mayo 2017]; 34(4): 156-165. Disponible en: http://scielo.isciii.es/scielo.php?script=sci_ arttext\&pid=S1130-05582012000400004\&lng=es. http://dx.doi.org/10.1016/j.maxilo.2012.02.002. 